\title{
Öffentliche und wissenschaftliche Bibliotheken
}

\author{
Tim Freytag und Michael Hoyler
}

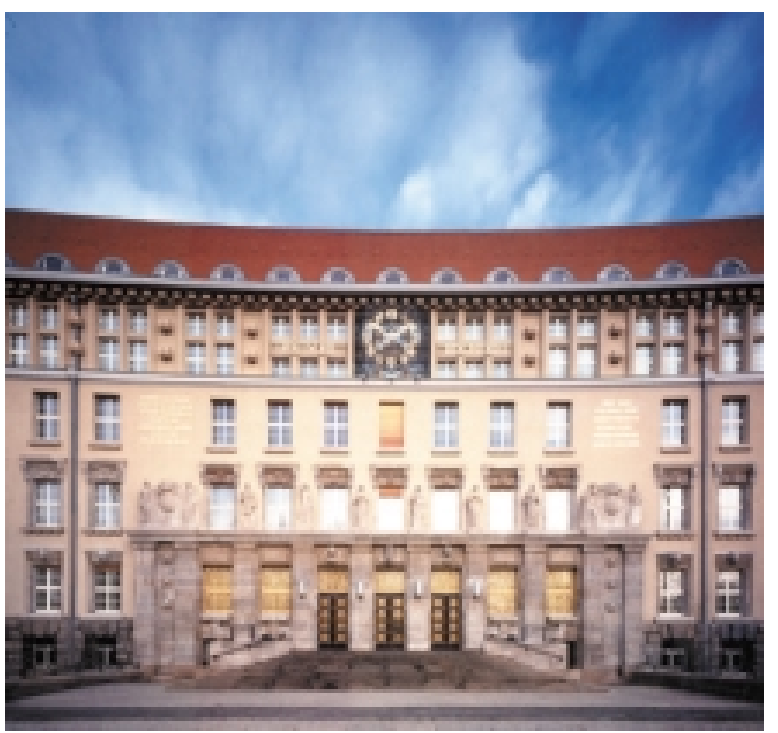

Bibliotheken sind ein wichtiges Bindeglied in der Archivierung und Weitergabe von Wissen und Kultur. Ihre Aufgabe ist die Verwaltung, Dokumentation und Bereitstellung von Büchern, Zeitschriften und anderen Medienbeständen. Darüber hinaus sehen sie sich als moderne Dienstleistungs- und Kompetenzzentren für alle Belange der Infor-

\section{Subsidiaritätsprinzip - rechtlicher} Grundsatz dafür, dass eine gesellschaftliche oder staatliche Aufgabe soweit möglich von der jeweils unteren (kleineren) Einheit wahrgenommen werden soll zentralörtliche Kriterien - Ausstattungsmerkmale, die für die Einstufung als zentraler Ort ausschlaggebend sind. Das Zentrale-Orte-System bezeichnet die von den jeweiligen Landesplanungen ausgewiesene hierarchische Gliederung des Siedlungssystems, die Städten unterschiedlicher Hierarchiestufen verschiedene Versorgungsfunktionen für das Umland zuweist.
Deutsche Bücherei Leipzig

mationsversorgung. In Deutschland gibt es rund 19.000 Bibliotheken unterschiedlicher Trägerschaft, Größe und Ausrichtung. Grundsätzlich werden öffentliche Bibliotheken, wissenschaftliche Bibliotheken und wissenschaftliche Spezialbibliotheken voneinander unterschieden.

Aufgrund der föderalen politischen Bundesrepublik nicht einheitlich organisiert. Die Kulturhoheit der Länder und das geltende Subsidiaritäts prinzip bewirken eine dezentrale $\mathrm{Zu}$ ständigkeit auf der Ebene der Länder und Kommunen mit einer entsprechend großen Vielfalt im Medienbestand und in der Qualität der Bibliotheken. Nach der Wiedervereinigung wurde die zentralistische Bibliotheksstruktur der DDR an das westdeutsche Bibliothekswesen angeglichen. In Anknüp fung an den Bibliotheksplan von 1973 legt das Planungspapier Bibliotheken '93 die Aufgabenverteilung im Bibliothekswesen nach zentralörtlichen Kriterien fest 2

Wissenschaftliche Bibliotheken Wissenschaftliche Bibliotheken 3 orientieren sich in ihrem Angebot vor allem an den Bedürfnissen von Forschung, Studium und Lehre. Die Nutzung ist in der Regel öffentlich. Den größten Bestand weist die 1990 aus den Vorgängerinstitutionen der beiden deutschen Staaten zusammengeschlossene Die Deutsche Bibliothek auf. Mit Hauptsitz in Frankfurt am Main (Deutsche Bibliothek, seit 1946) und ihren weiteren Standorten in Leipzig (Deutsche Bücherei, seit 1912 Foto) und
Berlin (Deutsches Musikarchiv, seit 1970) ist Die Deutsche Bibliothek die zentrale Archivbibliothek mit Pflicht exemplarrecht sowie das nationalbibliographische Zentrum der Bundesrepublik und erfüllt damit die Funktion einer Nationalbibliothek. Ähnliche Bedeutung besitzen die Staatsbibliothek zu Berlin - Preußischer Kulturbesitz und die Bayerische Staatsbibliothek in Mün chen als zentrale Universalbibliotheken mit einem Bestand von 9,4 bzw. 7,2 Mio. Büchern. Auf nationaler Ebene unterstützen sie Die Deutsche Biblio thek durch Zusammenarbeit in wichti gen Bereichen des Bibliothekswesens.

Weiterhin bestehen Regionalbibliotheken, die häufig unter der Bezeichnung Staats- oder Landesbibliothek als wissenschaftliche Universalbibliotheken in der Tradition ehemaliger Fürsten-, Hof- oder Stadtbibliotheken geführt werden. Hinzu kommen 79 Universitätsbibliotheken und etwa doppel so viele sonstige Hochschulbibliotheken, die in der Regel vom Land getragen und an zahlreichen Standorten um verschiedene Seminar-, Instituts- oder biet möglichst vollständig zu sammeln und im Leihverkehr zur Verfügung zu stellen. Darüber hinaus bestehen für Wirtschaftswissenschaften, Medizin, Technik und deren Grundlagenwissenschaften sowie Landbau vier zentrale Fachbibliotheken.

Unter dem Begriff wissenschaftliche Spezialbibliotheken werden mehrere tausend meist stark spezialisierte Bibliotheken in öffentlicher oder private Trägerschaft gefasst, wie etwa die Bibliotheken von Firmen, Museen oder Behörden. Diese sehr heterogene Gruppe verbindet die konsequente Ausrichtung auf bestimmte Sammelgebiete, auch wenn die einzelnen Einrichtungen hin sichtlich des Bestandsumfangs sowie de finanziellen und räumlichen Situation erheblich variieren.

Leihverkehr und Verbundsysteme

Die Zusammenarbeit von Bibliotheken im Leihverkehr ermöglicht es dem Benutzer, Medien einer auswärtigen Bibliothek über die ansässige Bibliothek zu bestellen und einzusehen. Die Organisa

\section{Funktionsstufen von Bibliotheken}

\begin{tabular}{|c|c|c|}
\hline Stufe & Literaturbedarf & Art der Bibliothek \\
\hline 1 & $\begin{array}{l}\text { Grundbedarf } \\
\text { erweiterter } \\
\text { Grundbedarf }\end{array}$ & $\begin{array}{l}\text { kleinere und mittlere öffentliche Bibliotheken } \\
\text { Fahrbibliotheken } \\
\text { Mittelpunktbibliotheken, Zweigstellen der Großstadtbibliotheken }\end{array}$ \\
\hline 2 & gehobener Bedarf & Zentralbibliotheken großstädtischer Bibliothekssysteme \\
\hline 3 & spezialisierter Bedarf & $\begin{array}{c}\text { Landesbibliotheken } \\
\text { Hochschulbibliotheken } \\
\text { Spezialbibliotheken } \\
\text { Großstadtbibliotheken (ab } 400000 \text { Einwohner) }\end{array}$ \\
\hline 4 & $\begin{array}{l}\text { hochspezialisierter } \\
\text { Bedarf }\end{array}$ & $\begin{array}{c}\text { Staatsbibliotheken } \\
\text { Zentrale Fachbibliotheken } \\
\text { Universitäts- und Staatsbibliotheken mit Sondersammelgebieten } \\
\text { einige Spezialbibliotheken }\end{array}$ \\
\hline
\end{tabular}

Klinikbibliotheken ergänzt werden. Unter den Universitätsbibliotheken besitzt die Bibliothek der Humboldt-Universität zu Berlin mit 5,8 Mio. Exemplaren den größten Buchbestand. Eine besondere fachbezogene Spezialisierung erfolgt an einzelnen Bibliotheken in Form von Sammelschwerpunkten nach einem von der Deutschen Forschungsgemeinschaft (DFG) laufend geförderten Plan, der die Bibliotheken verpflichtet, die deutsche und ausländische wissenschaftliche Literatur auf dem jeweiligen Ge- tion des Leihverkehrs sieht einen Austausch auf internationaler, nationaler, regionaler und lokaler Ebene vor. Zur Durchführung des überregionalen Leihverkehrs (Deutscher Leihverkehr) sind zehn Leihverkehrsregionen ausgewiesen. In ihren Grenzen orientieren sie sich zum Teil an den Ländern. Im Norden werden Bremen, Hamburg, Schleswig-Holstein und Mecklenburg-Vorpommern zu einer gemeinsamen Region zusammengefasst. Weiterhin kooperieren Berlin und Brandenburg. Nord- 


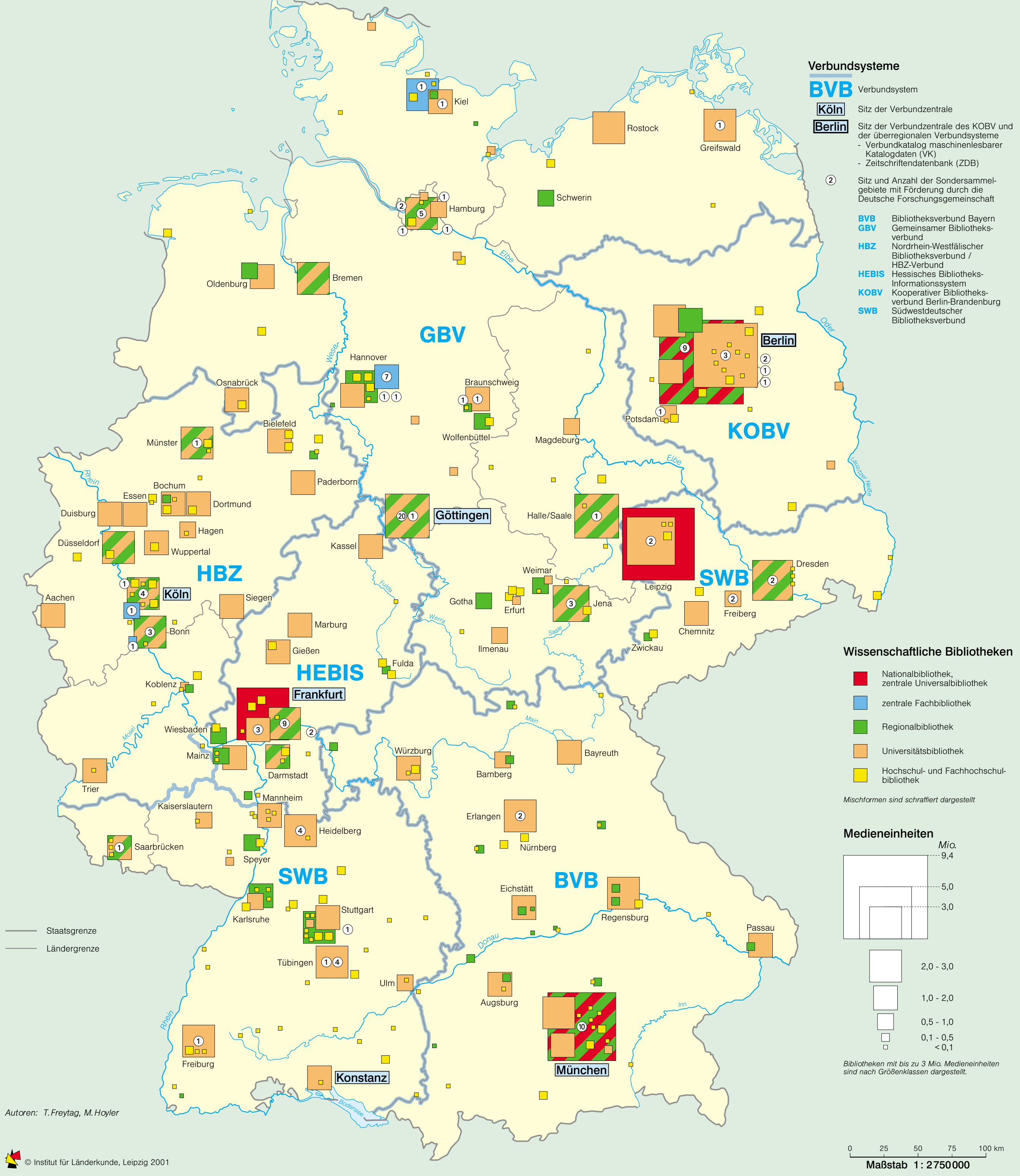


(4) Entwicklung öffentlicher Bibliotheken 1991-1998 auf Bundesebene und nach Ländern
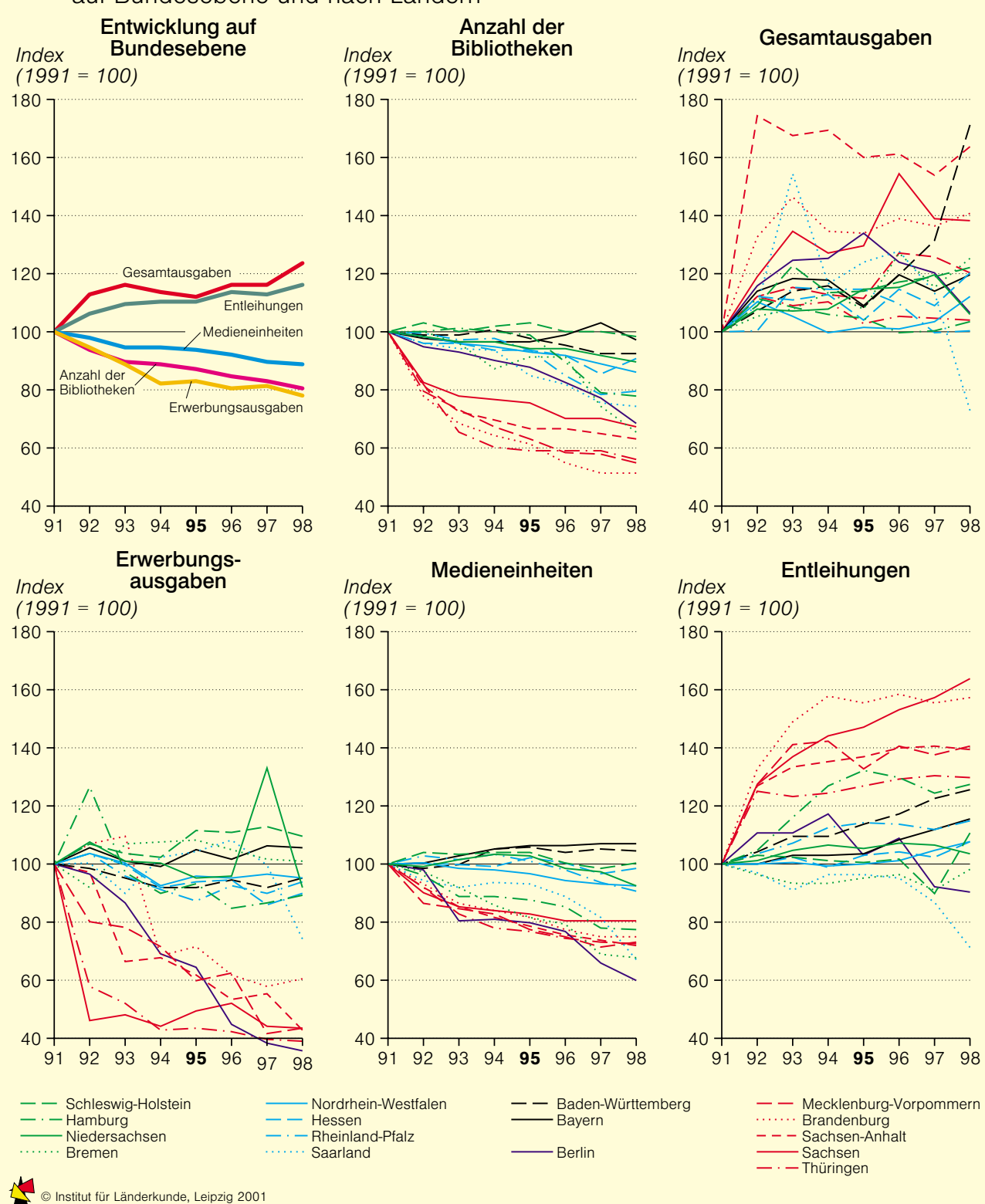

rhein-Westfalen, Hessen und BadenWürttemberg bilden jeweils mit einem Teil von Rheinland-Pfalz eigene Leihverkehrsregionen, wobei das Saarland der Leihverkehrsregion von BadenWürttemberg angehört.

Im Zuge der wachsenden Bedeutung elektronischer Datenverarbeitung im Bibliothekswesen wurden die Medienbestände in den vergangenen Jahren zu einem großen Teil elektronisch katalogisiert. Diese Daten werden in sechs regionalen Verbundsystemen zusammengeführt 3 und stehen im Internet für Recherchezwecke zur Verfügung. Dadurch wird das Verfahren einer Abwicklung von Fernleihen über die Zentralkataloge der Leihverkehrsregionen zunehmend durch Direktbestellungen bei den entsprechenden Bibliotheken ersetzt. Um den stark ansteigenden Leihverkehr effizienter zu gestalten, gewinnen örtliche und überregionale Dokumentlieferdienste an Bedeutung, die sich unter Verwendung neuer Medien auf die schnelle Beschaffung von Bü-

chern und Zeitschriftenartikeln speziali sieren, wie z.B. die Bund-Länder-Initiative zur Beschleunigung der Literaturund Informationsdienste SUBITO.

\section{Öffentliche Bibliotheken}

Zunächst stark einer erzieherisch-bildenden Tradition verhaftet, orientieren sich öffentliche Bibliotheken heute zunehmend am Leitbild eines modernen Kommunikations- und Informationszen trums. Neben Bestandspräsentation und Informationsversorgung leisten sie vielerorts auch durch soziale Bibliotheksarbeit und kulturelle Veranstaltun gen eine wichtige gesellschaftliche Aufgabe. Die überwiegend kommunale Finanzierung dieser Einrichtungen be wirkt allerdings erhebliche Unterschiede bezüglich Ausstattung und Nutzungs möglichkeiten. Von den etwa 13.000 ö fentlichen Bibliotheken sind rund 4000 mit hauptamtlich geschultem Personal besetzt (1. Diese zählen zur kulturellen Infrastruktur aller Großstädte mit mehr als 100.000 Einwohnern und sind in etwa $90 \%$ der Kommunen mit mehr als 20.000 Einwohnern, aber nur in weniger als 50\% der Gemeinden mit einer Einwohnerzahl zwischen 5000 und 20.000 vertreten. Mehr als ein Drittel der öffentlichen Bibliotheken steht in kirchlicher, überwiegend katholischer Trägerschaft und leistet häufig die Grundversorgung kleinerer Gemeinden.
Während der 1990er Jahre ist die Zahl der öffentlichen Bibliotheken in Deutschland merklich zurückgegangen 4. Dieser Prozess vollzieht sich vor dem Hintergrund einer in vielen Gemeinden angespannten finanziellen Situation und im Kontext der Anpassung des hierarchisch gegliederten Bibliothekssystems der DDR mit einer vergleichsweise hohen Bibliotheksdichte an eine westdeut sche Struktur, die eine überwiegen kommunale Selbstverwaltung dieser Einrichtungen vorsieht. Der wegen SchlieBungen zunehmend problematischen Versorgungssituation in ländlichen $\mathrm{Ge}$ bieten wird zum Teil mit dem Einsatz von Fahrbibliotheken begegnet, die auch in den Außenbezirken von Groß- und Mittelstädten Verwendung finden.

Bestand, Erwerb und Nutzung Der Medienbestand öffentlicher Bibliotheken umfasst neben Büchern und Zeitschriften auch Tonträger, Spiele und sonstige Materialien. Zur Grundversorgung der Bevölkerung wird in Gemeinden mit mehr als 5000 Einwoh- nern ein Mindestbestand von zwei Medieneinheiten je Einwohner angestrebt. Vergleichsweise günstig erscheint die Situation in den Stadtkreisen und im östlichen Teil Deutschlands, während in zahlreichen Landkreisen Westdeutschlands der Bestand weniger als eine Medieneinheit je Einwohner beträgt 5 . Insgesamt ist der Medienbestand der öf fentlichen Bibliotheken während der 1990er Jahre rückläufig. Der Bestandsabbau durch Verlust, Veräußerung u.a. ist sichtlich größer als die Zahl der Neuerwerbungen. Lediglich Bayern und Baden-Württemberg verzeichnen in diesem Zeitraum einen leichten $\mathrm{Zu}$ wachs 4.

Auch die Erwerbungsausgaben haben sich seit 1991 verringert. Dies gilt in

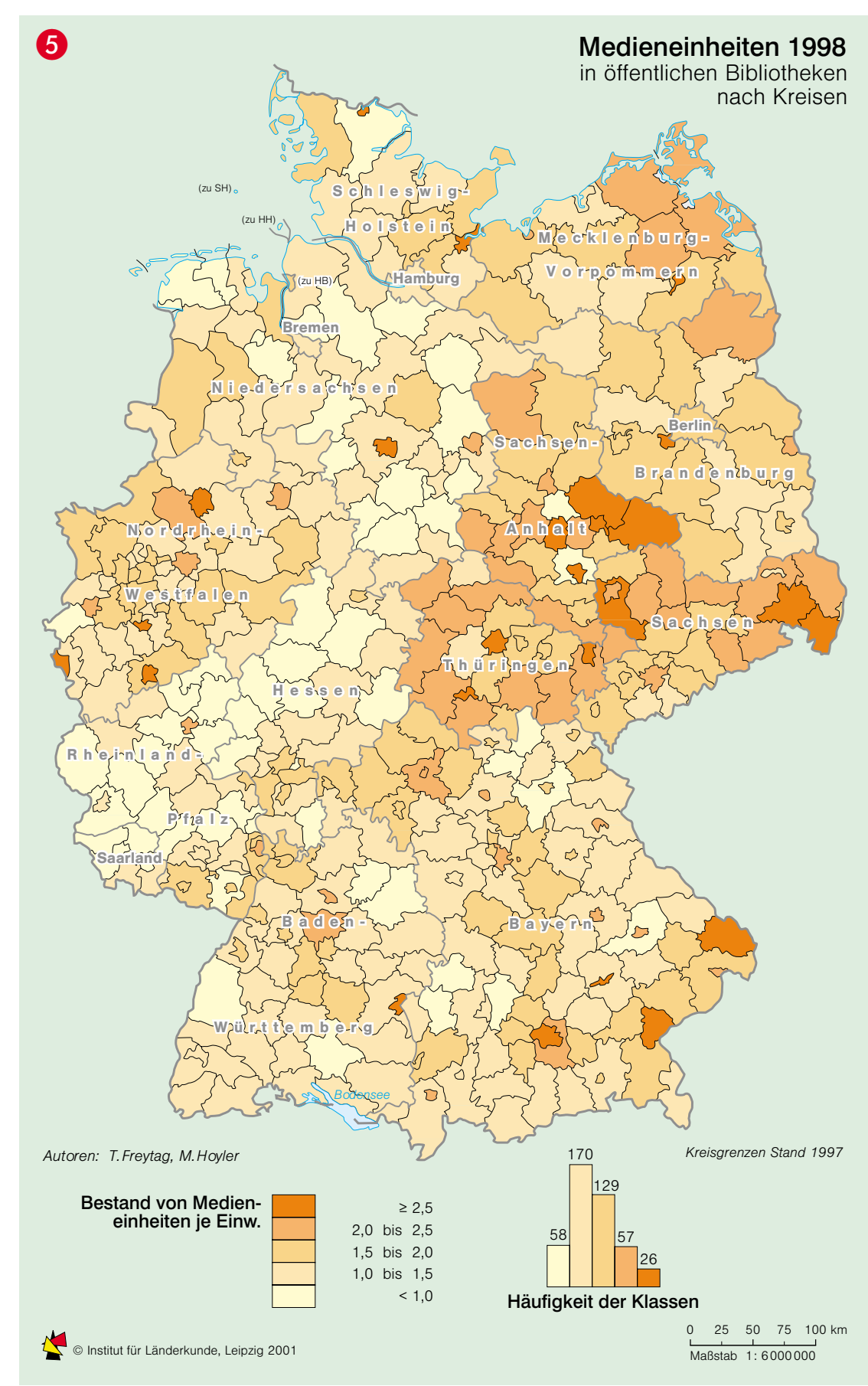


besonderer Weise für Ostdeutschland, das unmittelbar nach der Wiedervereinigung durch die Bereitstellung zeitlich begrenzter Fördermittel des Bundes und der Länder eine Modernisierung des Medienbestands vornehmen konnte 6 Die Erwerbungsausgaben je Einwohner liegen 1998 im Bundesdurchschnitt bei 2,07 DM und betragen damit ein knappes Neuntel der Gesamtausgaben.

Die Zahl der Entleihungen hat dagegen in den 1990er Jahren kontinuierlich zugenommen 4 und ist zwischen 1991 und 1998 von 3,5 auf 4 Entleihungen pro Einwohner gestiegen, wobei der vorhandene Medienbestand 2,5 mal pro Jahr umgesetzt wird. In regionaler Differenzierung verzeichnen die angrenzenden Gebiete Nordhessen, RheinlandPfalz und Saarland ein stark unter durchschnittliches Leihverhalten 7 Ähnlich geringe Werte lassen sich dort für Medienbestand und Erwerbungsausgaben beobachten. Zusammenfassen betrachtet sind die vorhandenen Dispa ritäten nicht allein Ergebnis der jeweil gen Siedlungs- und Wirtschaftsstruktur, sondern müssen unter Berücksichtigung aktueller kommunalpolitischer Entscheidungen als historisch gewachsene Bibliothekslandschaften verstanden werden.

\section{Perspektiven}

Das deutsche Bibliothekswesen zeigt aufgrund seiner dezentralen Organisationsstruktur ein heterogenes Bild. In den 1990er Jahren sehen sich Bibliotheken mit drei großen Herausforderungen konfrontiert: mit der Expansion neuer Informationstechnologien und entsprechender Medien, mit einer Verknappung der finanziellen Mittel bei gleichzeitigem Anstieg der Buchproduktion und mit der Transformation des ostdeut schen Bibliothekssystems.

Öffentliche Bibliotheken vollziehen einen Umstrukturierungsprozess, der ihre Rolle als Vermittler von Information, Bildung und Unterhaltung im Zeitalter neuer Medien neu definiert und sie zunehmend zu Orten der Kommunikation und Begegnung werden lässt.

Viele Kommunen entschließen sich auf-
Die Deutsche Bibliothek, Frankfurt a.M.

grund einer angespannten Haushaltslage zur Kostenbeteiligung der Bibliotheksbenutzer, eine Privatisierung öffentlicher Bibliotheken bildet bislang jedoch die Ausnahme.

Auch wissenschaftliche Bibliotheken sehen sich einer stark anwachsenden Zahl von Publikationen und den rapiden Entwicklungen im Bereich neuer Medien ausgesetzt. Hinzu kommen dras tische Preissteigerungen, die in beson derer Weise das Abonnement von Fachzeitschriften betreffen. Um langfristig international konkurrenzfähig zu bleiben, wird die Zusammenarbeit und Ver netzung der Bibliotheken unter Einsatz moderner Kommunikationstechnologien immer wichtiger. Zugleich müssen wissenschaftliche Bibliotheken die Be

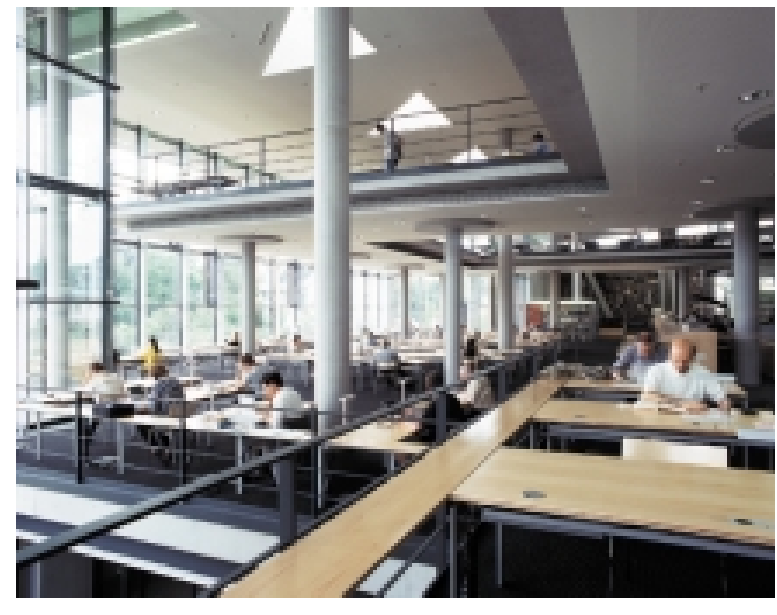

reitstellung, Bewahrung und Pflege ihres Medienbestands einschließlich wert voller Altbestände gewährleisten.*
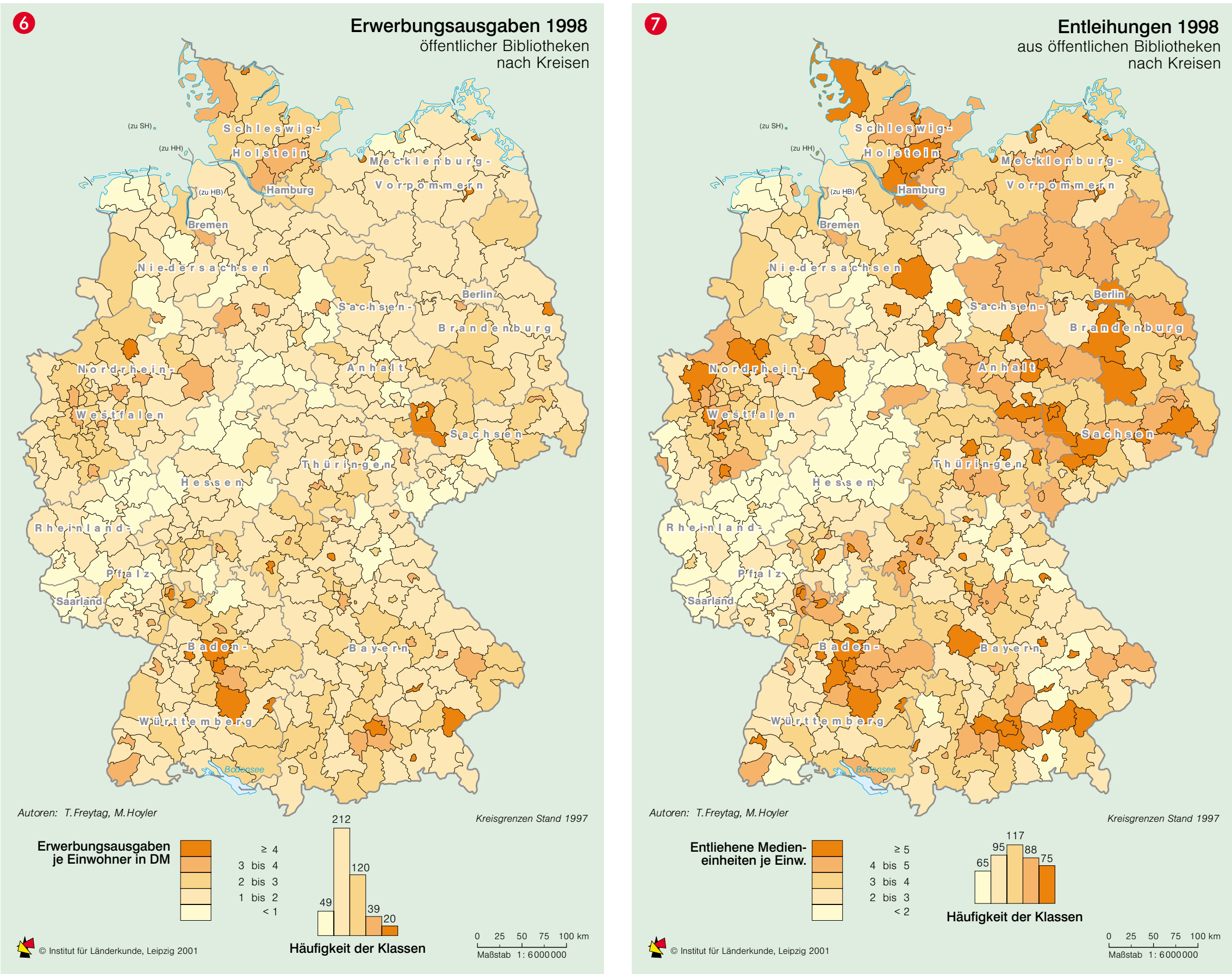